\title{
Review Article \\ Emerging Glycolysis Targeting and Drug Discovery from Chinese Medicine in Cancer Therapy
}

\author{
Zhiyu Wang, Neng Wang, Jianping Chen, and Jiangang Shen \\ School of Chinese Medicine, The University of Hong Kong, Estates Building, 10 Sassoon Road, Hong Kong \\ Correspondence should be addressed to Jianping Chen, jpjpchen@yahoo.com and Jiangang Shen, shenjg@hkucc.hku.hk
}

Received 31 December 2011; Revised 28 May 2012; Accepted 12 June 2012

Academic Editor: Francesca Borrelli

Copyright ( $) 2012$ Zhiyu Wang et al. This is an open access article distributed under the Creative Commons Attribution License, which permits unrestricted use, distribution, and reproduction in any medium, provided the original work is properly cited.

Molecular-targeted therapy has been developed for cancer chemoprevention and treatment. Cancer cells have different metabolic properties from normal cells. Normal cells mostly rely upon the process of mitochondrial oxidative phosphorylation to produce energy whereas cancer cells have developed an altered metabolism that allows them to sustain higher proliferation rates. Cancer cells could predominantly produce energy by glycolysis even in the presence of oxygen. This alternative metabolic characteristic is known as the "Warburg Effect." Although the exact mechanisms underlying the Warburg effect are unclear, recent progress indicates that glycolytic pathway of cancer cells could be a critical target for drug discovery. With a long history in cancer treatment, traditional Chinese medicine (TCM) is recognized as a valuable source for seeking bioactive anticancer compounds. A great progress has been made to identify active compounds from herbal medicine targeting on glycolysis for cancer treatment. Herein, we provide an overall picture of the current understanding of the molecular targets in the cancer glycolytic pathway and reviewed active compounds from Chinese herbal medicine with the potentials to inhibit the metabolic targets for cancer treatment. Combination of TCM with conventional therapies will provide an attractive strategy for improving clinical outcome in cancer treatment.

\section{Introduction}

Cancer is the second leading cause of mortality in human diseases worldwide. According to a national statistic report on the incidence and mortality in the USA, there were a total of $1,529,560$ new cancer cases and 569,490 deaths from cancer occurring in 2010 [1]. Surgery, chemotherapy, and radiotherapy, either alone or in combination, have been considered as conventional strategies for cancer treatment in the last century. With the rapid development of molecular medicine, novel therapeutic approaches, such as immunotherapy, molecular targeted therapy, and hormonal therapy, have been proposed to improve clinical outcomes for cancer patients [2-4]. However, those therapeutic approaches are not always effective and clinical outcome in survival rates is still poor. One of the major problems is that cancer cells gradually develop resistances to those therapies. Seeking for new therapeutic approaches to improve outcome of cancer treatment is timely important.
Complementary and alternative medicine (CAM) attracts much attention for drug discovery in current cancer research $[5,6]$. Among CAM modalities, TCM is particularly appreciated in both rural and well-developed urban areas of China based on its 5000-year-old history and a well-established theoretical approach $[7,8]$. In China, Chinese herbal medicine is widely used as an adjunct therapy to reduce the resistances and side effects of cancer cells to chemotherapy and radiotherapy. Chinese herbal medicine in combination with chemotherapy and radiotherapy potentially improves clinical outcome in cancer treatment [9]. However, the relatively poor designs in many clinical reports, such as lack of quality standardization of herbal products, shortage of well-designed randomized controlled trials (RCT), and the limited sample size, bring difficulty to evaluate the benefits or disadvantages of herbal medicine for cancer treatment $[10,11]$. TCM approaches have always been met with much skepticism and pessimism by the West. In fact, medicinal herbs are very important 
resources for drug discovery in cancer treatment. The direct experience from TCM on human subjects and its long history provide important cues for drug development. Of the 121 prescription drugs in use today for cancer treatment, 90 are originally derived from medicinal plants. Almost $74 \%$ of those drugs were discovered from folk medicine $[12,13]$. In a previous review article, 48 out of 65 new drugs approved for cancer treatment during 1981-2002 were natural products, leading from natural products, or mimicked natural products in one form or another [14]. Among the drugs, the most well-known examples include Vinca alkaloids (vincristine, vinblastine, vindesine, vinorelbine), taxanes (paclitaxel, docetaxel), podophyllotoxin and its derivative (etoposide, teniposide), camptothecin and its derivatives (topothecan, irinothecan), anthracyclines (doxorubicin, daunorubicin, epirubicin, idarubicin), and others [15]. There is an impressive revival of seeking natural lead compounds for the generation of semisynthetic derivatives. Current progress in this aspect not only provide a chemical bank from natural sources for drug discovery but also bring better understanding for the chemical basis of Chinese herbal medicine for cancer treatment.

With its multiple components, TCM formulas and therapies are generally considered to regulate multiple cellular signal pathways [16]. Herbal medicine and their active components are promising sources for the designs of more effective and less toxic agents in cancer chemoprevention and treatment [17]. Many TCM products or single active components have been reported to inhibit a variety of processes in cancer cell growth, invasion, and metastasis by modulating a wide range of molecular targets, including cyclooxygenase-2 (COX-2), nuclear factor kappa B (NF$\kappa \mathrm{B})$, and nuclear factor erythroid 2-related factor 2 (Nrf2)mediated antioxidant signaling pathways. A previous review article summarized the therapeutic targets of traditional Ayurvedic medicine for inflammation and cancer. The targets include growth factor signaling (e.g., epidermal growth factor); prostaglandin (e.g., COX-2); inflammation factors (e.g., inflammatory cytokines: TNF, IL-1, IL-6, chemokines); drug resistance genes (e.g., multidrug resistance); cell cycle proteins (e.g., cyclin D1 and cyclin E); angiogenesis factors (e.g., vascular endothelial growth factor); invasion mediators (e.g., matrix metalloproteinases); apoptosis related genes (e.g., bcl-2, bcl-X(L), XIAP, survivin, FLIP); proliferation factors (e.g., c-myc, AP-1, growth factors), [18]. With the development of systematic biology and bioinformatics, more attention has been paid to the synergistic effects of herbal medicine on "common" signal pathways involved in the proliferation, invasion, metastasis, and apoptosis of cancer cells. It is interesting to ask the question whether herbal medicine and it's derivatives can specially target on tumor biomarkers and affect survival of cancer cells.

Molecular targeted therapy has been attracted much attention in cancer treatment [19]. Ideally, the identified targets should be preferentially expressed or activated in cancer cells but not in normal cells. Combining molecular and genetic technologies, a number of small molecular inhibitors and antibodies targeting on kinases or oncogenes has been designed and synthesized [20, 21]. The most well-known examples include the small molecule Gleevec (targeting on BCR-ABL translocation associated with chronic myelogenous leukemia), and antibody-based molecule Herceptin (c-erbB-2 overexpression related to breast cancer), [22, 23]. However, primary and secondary resistances to these targeted molecules severely reduce their therapeutic efficacy $[24,25]$. Therefore, seeking more distinctive molecular targets and their corresponding drug candidates become important tasks for oncologists.

Cancer cells can be distinguished from normal cells in several hallmarks. One of hall marks is that cancer cells have a fundamentally different bioenergetic metabolism from that of nonneoplastic cells. In normal cells, energetic metabolism mostly relies upon the process of mitochondrial oxidative phosphorylation which consumes glucose and oxygen to produce energy. In contrast, cancer cells have developed an altered metabolism that allows them to sustain higher proliferation rates [26]. Cancer cells could predominantly produce energy by glycolysis followed by lactic acid fermentation, even in the presence of oxygen-this is known as the "Warburg Effect" [27, 28]. Cancer glycolysis is a critical step in carcinogenesis and oncogenic activation [29, 30]. Targeting on glycolysis becomes an attractive strategy in cancer diagnosis and treatment clinically [31]. The inhibitors targeting some key enzymes showed promising anticancer effects and have been approved for clinical trials [32]. Chinese herbal medicine could specifically target on the molecules in the metabolic pathways of cancer. Recent progress leads to the discoveries of many active compounds derived from Chinese herbs with the properties of inhibiting cancer cell glycolysis activity. Therefore, in the present paper, we intend to review current progress about TCM-derived phytochemicals which specifically target the key enzymes and proteins involved in cancer glycolysis.

\section{Glycolytic Pathway as a Target for Cancer Therapy}

Otto Heinrich Warburg, a pioneer in the study of respiration, made a striking discovery in the 1920s from extensive observation on the metabolic behavior of cancer cells. Even in the presence of oxygen, cancer cells prefer to metabolize glucose by glycolysis, a less efficient pathway for producing ATP [33]. The respiratory behavior was subsequently demonstrated in a various kinds of cancer cells and was called aerobic glycolysis [34-36]. The exact reasons why tumor cells exhibit elevated glycolysis and use this primitive and less energy-efficient pathway to generate ATP is still unclear. Accumulating evidences have suggested multiple mechanisms contributing to the unique phenomenon: (1) mitochondrial DNA mutations; (2) nuclear DNA mutations; (3) oncogenic transformation; (4) influences of the tumor microenvironment [37-39]. All these factors result in mitochondrial dysfunction and make cancer cells generate ATP much more dependently on the glycolytic pathway. Given the mitochondrial respiratory abnormality, cancer cells have to uptake much more glucose to produce enough 
ATP supporting rapid proliferation needs. At present, the phenomenon has been exploited clinically for the detection of tumors by fluorodeoxyglucose positron emission tomography (FDG-PET) [40]. Inhibition of aerobic glycolysis becomes an important strategy to preferentially kill cancer cells and to find anticancer agents based on Warburg hypothesis [41, 42]. As illustrated in Figure 1, cancer cells in the tumor mass could be divided into oxygenated and hypoxic cells. Hypoxic cancer cells predominately depend on glycolysis to produce energy. The glycolytic pathway is a series of metabolic reactions catalyzed by multiple enzymes or enzyme complexes. From the original glucose uptake to the final lactate production, the key steps include: (1) the increasing uptake of glucose by elevated expression of glucose transporter-1 (GLUT1) and sodium glucose cotransporter-1 (SGLT1); (2) active ATP generation reaction by upregulation of phosphoglycerate kinase (PGK) and pyruvate kinase (PK); (3) regeneration of $\mathrm{NAD}^{+}$by lactate dehydrogenase $(\mathrm{LDH})$; (4) out-transport and reuptake of lactate by monocarboxylate transporter (MCT), mainly MCT1 and MCT4 $[43,44]$. In oxygenated cancer cells, the reuptaken lactate could be metabolized to pyruvate and reentered the mitochondrial tricarboxylic acid cycle to produce ATP. Each reaction in the glycolytic pathway is activated by a specific enzyme or enzyme complex. Interrupting any of the above proteins could lead to metabolism blockade followed by cell death. The activities of many enzymes in the pathway are controlled by two factors including c-myc and hypoxia inducible factor$1 \alpha(\mathrm{HIF}-1 \alpha)[45,46]$. Many studies have demonstrated an increase in the activities of the glycolytic enzymes such as hexokinase, lactate dehydrogenase A (LDH-A), and glyceraldehydes-3-phosphate dehydrogenase (GAPDH) in various types of tumors and cancer cell lines [47-49]. In addition, silencing of these overexpressed enzymes, such as LDH-A or pyruvate kinase (PKM2), has been documented efficiency for inhibiting cancer cell proliferation, inducing apoptosis and reversing multidrug resistance [50-52]. Furthermore, some glycolytic enzymes are multifunctional proteins. For example, hexokinase and enolase play critical roles in transcription regulation [53, 54], while glucose-6phosphate isomerase may affect cell motility [55]. Therefore, developing novel glycolytic inhibitors is an important direction in current cancer research. As TCM has held an important position in primary health care in China and been recently recognized by the West as a fertile source for revealing novel lead molecules for modern drug discovery, more and more herb-derived bioactive compounds have been identified for cancer therapy. Among them, several have been proved to be effective in suppressing cancer glycolytic activity by targeting on particular enzymes. Herein, we review current evidence on the studies of herbal medicine related to regulate several key enzymes in the glycolytic pathway including HIF- $1 \alpha$, hexokinase, and LDH-A.

\section{Glycolytic Molecular Targets and Herb-derived Inhibitors}

3.1. HIF-1 $\alpha$. HIF-1 is a basic helix-loop-helix heterodimeric transcriptional factor composed of $\alpha$ and $\beta$ subunits [56].
HIF-1 is overexpressed in various types of cancer, and the levels of its activity have already been demonstrated closely to tumorigenicity, angiogenesis and also glycolytic activity $[57,58]$. HIF- $1 \alpha$ levels are primarily induced by hypoxia, growth factors, and oncogenes. As shown in Figure 2, under normoxia, HIF- $1 \alpha$ is rapidly and continuously degraded by the ubiquitin-proteasome pathway. The prolyl hydroxylation of oxygen-dependent degradation domain (ODD) and binding with Von Hippel-Lindau (VHL) play a critical role in regulation of HIF- $1 \alpha$ degradation. However, under hypoxic condition, the absence of oxygen prevents the prolyl hydroxylase process, allowing HIF- $1 \alpha$ to accumulate and translocate to the nucleus, where it forms an active complex with HIF-1 $\beta$ and activates a series of downstream gene transcription $[59,60]$. Besides hypoxia, the expression of HIF-1 was also regulated by other factors including oncogenes ( $\mathrm{p} 53$, VHL, etc.), cytokines (EGF, TGF- $\alpha$, IGF1 , and -2 , etc.) and some posttranslational modifications including hydroxylation, ubiquitination, acetylation, and phosphorylation, $[61,62]$. A number of glycolytic related genes are regulated by HIF- $1 \alpha$, such as GLUT-1, hexokinase, LDH-A, and PDK1. Interruption of HIF- $1 \alpha$ signaling revealed to inhibit cancer growth in both in vitro and in vivo experimental models [63].

Recently, a remarkable progress has been made to seek selective HIF- $1 \alpha$ inhibitors for cancer treatment from herbal medicine. Apigenin, a plant flavonoid compound, is considered as a typical HIF- $1 \alpha$ inhibitor [64-72]. Apigenin is isolated from a traditional Chinese herb Apium graveolens var.dulce. Apigenin has been shown to inhibit proliferation and induce apoptosis in a wide range of malignant cells including breast, ovarian, prostate, and lung cancer, [65-68]. Apigenin suppressed tumor angiogenesis by downregulating VEGF, a proangiogenic protein regulated by HIF- $1 \alpha[69$, 70]. Apigenin inactivated the PI3K/Akt pathway in prostate cancer cells $[71,72]$. Apigenin reduced HIF- $1 \alpha$ stability and HIF- $1 \alpha$ mRNA expression in human prostate cancer PC3-M cells via PI3K/Akt/GSK-3 $\beta$ pathway [73]. Apigenin promoted HIF- $1 \alpha$ degradation via disrupting HIF- $1 \alpha$-Hsp 90 interaction under hypoxia [74]. Oral administration of apigenin resulted in tumor growth abrogation in prostate cancer xenografts, accompanied by inactivation of Akt, and induction of apoptosis [72]. Another study also revealed that apigenin in vivo administration significantly limited tumor growth and angiogenesis in both prostate and ovarian cancer models. Meanwhile, the expression of HIF- $1 \alpha$ and VEGF were also down-regulated in apigenin-treated tumor samples [69]. Chrysin, isolated from Oroxylum indicum (L.)Vent, mediated apoptosis in various types of cancer including prostate, thyroid, and leukemia malignancies [7577]. Chrysin significantly inhibited prostate cancer growth and angiogenesis with a decreased HIF- $1 \alpha$ expression [78]. The suppression of HIF- $1 \alpha$ expression could be related to different mechanisms including stimulation of $\mathrm{PHD}$ activity, disruption of HIF- $1 \alpha$-HSP90 interaction and direct inhibition of HIF- $1 \alpha$ protein synthesis [78]. Epigallocatechin gallate (EGCG) is a widely spread flavonoid in Chinese herbs. The anticancer effects of EGCG were well documented $[79,80]$. Many of its intracellular molecular targets, such 


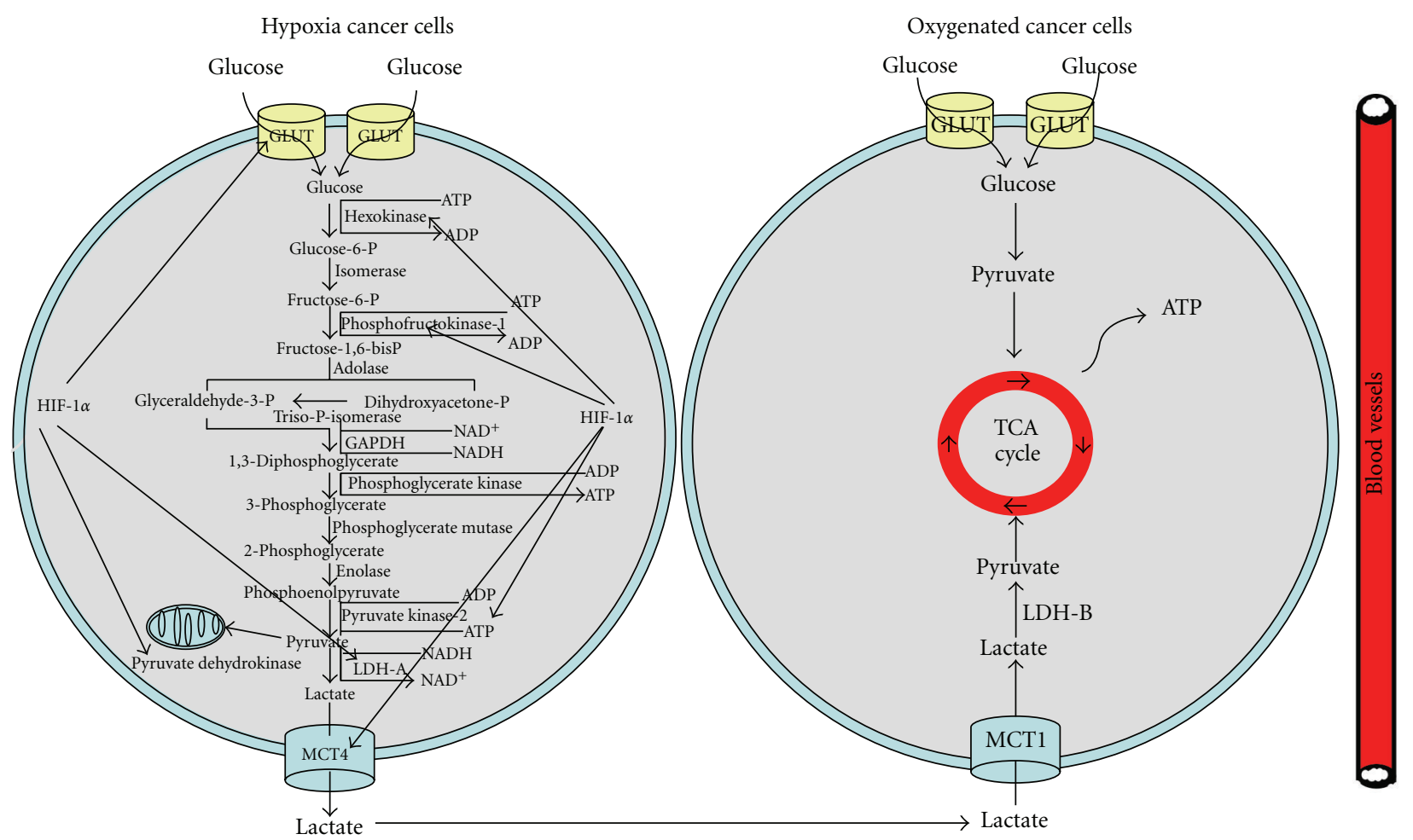

FIGURE 1: Glycolytic pathway and the role of HIF-1 $\alpha$ in regulating glycolysis. Glucose was uptaken by increased expression of GLUT in hypoxia cancer cells. Through a series of enzyme reaction, glucose was finally metabolized into lactate and ATP, NAD ${ }^{+}$was also regenerated by LDH-A for maintaining continuous glycolysis. Lactate was exhausted out of cancer cells by MCT4 and then uptaken by oxygenated cancer cells through MCT1. In the presence of oxygen, lactate is oxidized into pyruvate by LDH-B and pyruvate enters the tricarboxylic acid (TCA) cycle to produce ATP. HIF- $1 \alpha$ was the main regulator of some enzymes expression in the glycolytic pathway, including GLUT-1, hexokinase, phosphofructokinase, pyruvate kinase, pyruvate dehydrogenase, LDH-A, and MCT.

as proteasomes, MAP kinases, VEGF, erythropoietin, and glucose transporters, are directly or indirectly regulated by HIF- $1 \alpha[81,82]$. Several studies indicate that EGCG could inhibit HIF- $1 \alpha$ expression by both blocking PI3K/Akt signaling pathway and reducing interaction between Hsp90 and HIF- $1 \alpha$ [83]. Besides, curcumin, a well-validated anticancer compound extracted from Curcuma longa, has been found to interact directly with more than 30 different proteins including transcriptional factors (NF- $\kappa \mathrm{B}, \mathrm{AP}-1$, STAT, and $\beta$-catenin, etc.), growth factors and protein kinases (EGFR, ErbB-2, VEGF, EGF, MAPKs, and CXCR4 , etc.), inflammatory factors (TNF- $\alpha$, IL- $1 \beta$, IFN- $\gamma$, and COX-2, etc.), adhesion molecules (integrins, fibronectin, vitronectin, and collagen IV, etc.) and apoptosis-related proteins (death receptors, Bax, Bcl-2, and survivin, etc.) $[84,85]$. Several studies also demonstrated that curcumin dose-dependently inhibited HIF- $1 \alpha$ and HIF- $1 \beta$ gene at transcription level $[86,87]$. Using luciferase reporter gene assay, an Indian herb Ophiorrhiza trichocarpon was identified to have the strongest HIF- $1 \alpha$ inhibitive effects among more than 6,000 crude natural products. The extracts of Ophiorrhiza trichocarpon were shown to reduce hypoxiainduced HIF- $1 \alpha$ accumulation to $22 \%$ relative to the normal control. Following bioactivity-guided fractionation assay validated camptothecin to be the best HIF- $1 \alpha$ inhibitor among 84 fractions isolated from the medicinal plant [88]. Since the primary molecular target of camptothecin is established as human DNA topoisomerase I, its anticancer effects need to be further verified in animal experiments. Detail mechanisms in regulation of HIF- $1 \alpha$ transcription activity remain to be further investigated. Terpenoids were also reported to inhibit HIF- $1 \alpha$ activity. Nguyen et al. carried out screening assay for HIF- $1 \alpha$ inhibitors from Salvia miltiorrhiza extracts by using luciferase gene reporting system. Diterpenes including sibiriquinone A, sibiriquinone $B$, cryptotanshinone, and dihydrotanshinone were finally identified to be strong HIF-1 $\alpha$ inhibitors [89]. Although many herb-derived compounds are effective in suppressing HIF- $1 \alpha$ activity, little compound is identified to specifically bind to HIF-1 $\alpha$. The structure-activity relationship and chemical optimization study are important topics for further studies on this direction. In addition, as many studies were conducted with cell systems, in vivo animal experiments are essentials to verify the bioactivities of targeting on HIF$1 \alpha$ activity contributing to their anticancer effects for drug development. 


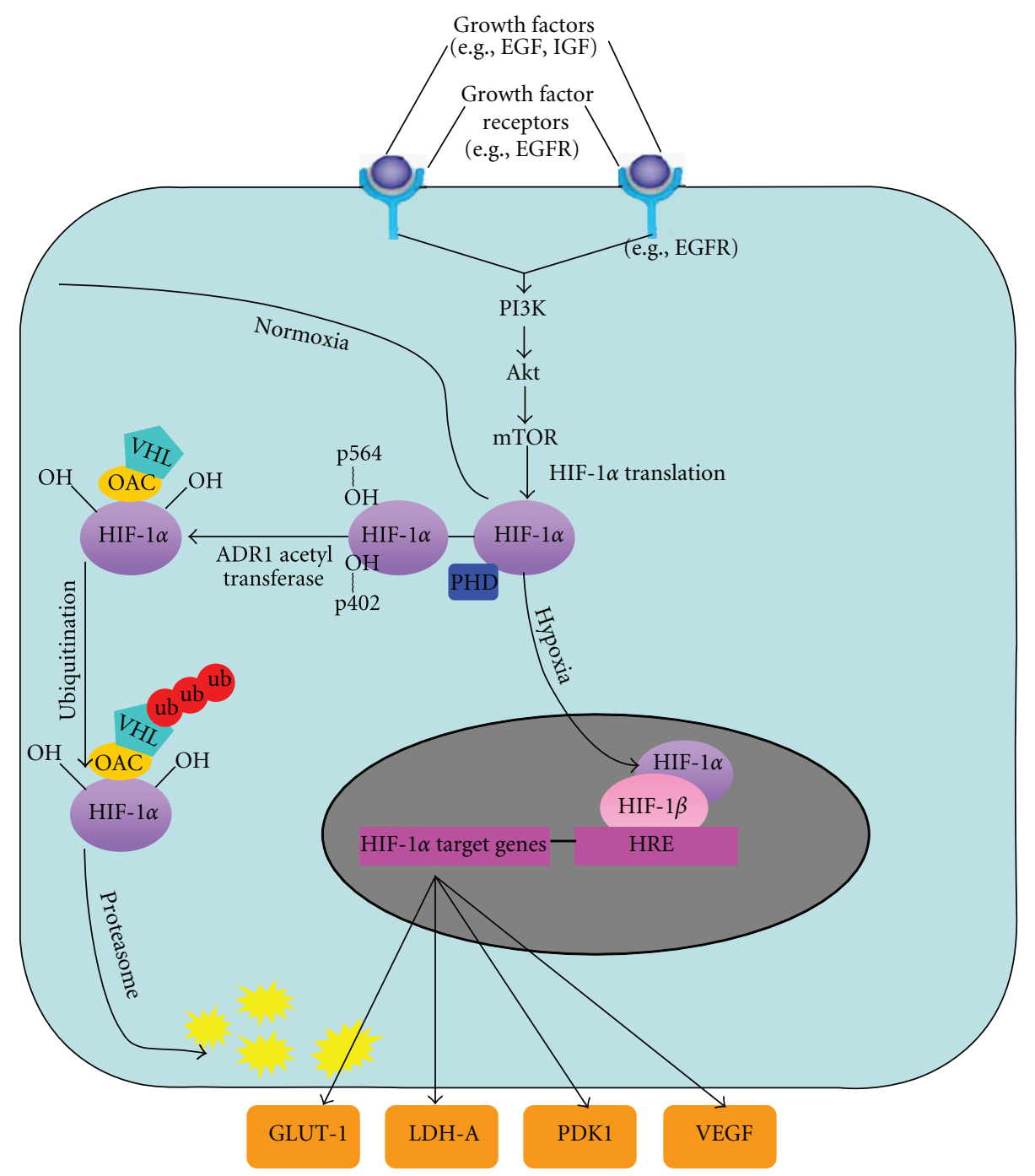

Figure 2: The intracellular HIF- $1 \alpha$ regulation pathway in normoxia and hypoxia. Under normoxia, HIF- $1 \alpha$ will be constituently ubiquitinated and subsequently degraded via proteasomal pathway after recruitment of von Hippel-Lindau protein (pVHL), which depends on the hydroxylation of proline residues on 564 and 402 . However, under hypoxia, the praline hydroxylation of HIF- $1 \alpha$ will be inhibited, HIF- $1 \alpha$ will be translocated into the nucleus and combine with HIF- $1 \beta$, then activate the transcription of a series of downstream genes including LDH-A, PDK1, GLUT-1, and VEGF. The levels of HIF- $1 \alpha$ were also influenced by the PI3K/AKT pathway after stimulation of growth factors such as EGF and IGF.

3.2. Hexokinase. Hexokinase (HK) controls the conversion of glucose to glucose-6-phosphate (G6P), which serves as the starting point for sugar to enter the glycolytic pathway or for glycogen synthesis [90]. Four isoforms of hexokinase have been identified in mammals, among which hexokinase II (HKII) is a major form responsible for maintaining the high glucose catabolic rates of malignant cells [91]. HKII overexpression was found in various types of cancers such as liver, breast, and lung cancers [92]. In addition to its glucose phosphorylation activity, HKII is capable of binding to the voltage-dependent anion channel (VDAC) on the mitochondrial outer membrane [93]. The specific binding not only allows efficient use of mitochondrialgenerated ATP served as glycolytic fuel, but also stabilizes the mitochondrial membrane and prevents the release of proapoptotic factors, such as cytochrome C [94]. Disrupting the interaction between HKII and VDAC could inhibit cell proliferation and induce apoptosis through decreasing ATP supply and destabilization of mitochondrial membranes (Figure 3). Therefore, developing inhibitors targeting HKII is an interesting topic in anticancer drug development. 2deoxyglucose (2-DG) and 3-bromopyruvate (3-BrpA) are well known HKII inhibitors [95, 96]. These pharmacological inhibitors have been proved to be effective in disrupting the binding of HKII to the mitochondrion, depleting ATP, inhibiting cell cycle progression, and inducing cell death. A TCM formula Ben Cao Xiao Ke Dan was revealed a strong inhibitory effect on HKII activity. However, exact 


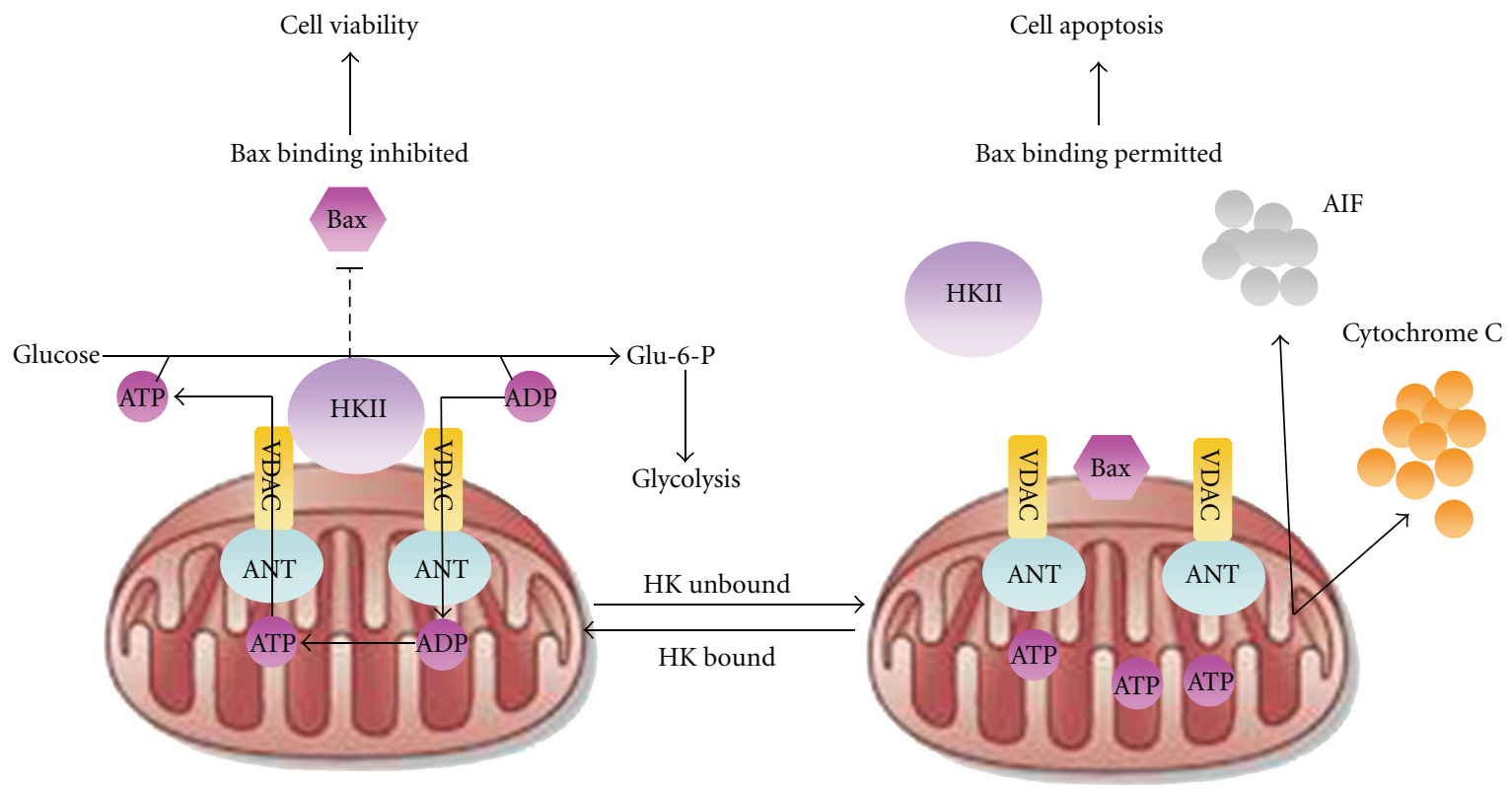

FIgURE 3: Antiapoptotic and metabolic roles for mitochondrial hexokinase II (HKII). (Left panel) Specific binding of HKII to the outer mitochondrial membrane (OMM) promotes ATP exchanges through complexes consisting of voltage-dependent anion channel (VDAC) and adenine nucleotide (ANT). The effluxed ATP could directly participate the transition from glucose to glucose-6-phosphate, which accelerates the glycolytic activity. Meanwhile, HKII binding to OMM also antagonizes Bax interaction with mitochondrial contact site, which prevents apoptosis occurrence; (Right panel) HKII unbound resulted in the "close" of VDAC-ANT channel, which induces Bax integration and potential changes between outer and inner mitochondrial membrane, and finally leading to release of cytochrome $\mathrm{C}$ and apoptosis-inducing factors (AIF) from mitochondrion.

phytochemicals in the formula accounting for this inhibitory effect remains unclear [97]. Methyl jasmonate, a plant lipid derivative, exists in many herbs and functions as a signaling molecule in the stress response. Methyl jasmonate was shown to induce apoptosis in various malignancies including prostate, cervical, and bladder cancers [98-100]. Recent studies found that its apoptosis-induction effects are closely correlated to the disruption of the interactions between HKII and VDAC [101]. Although some HKII inhibitors, such as 2-DG and 3-BrpA, were approved for clinical trials, the nonspecific inhibitions on all isoforms of HKs and normal cells might result in toxic effects when they are applied in patients. Therefore, to develop agents specifically targeting on HKII of cancer cells is a direction for further studies.

3.3. $L D H-A$. LDH-A is emerging as a novel therapeutic target in the glycolytic pathway. LDH has two subtypes: LDH-A, also called the skeletal muscle type or LDH-M, and LDH-B, also known as the heart type or LDH-H. LDH-A exhibits kinetic features suitable for conversion of pyruvate into lactate, whereas LDH-B has kinetic features suitable for conversion of lactate into pyruvate. LDH-A is an attractive target for cancer therapy because its expression is largely confined to skeletal muscle [102]. Moreover, human subjects with LDH-A deficiency show myoglobinuria under intense anaerobic exercise, and individuals with complete lack of LDH-A subunit have been documented with no apparent increase in hemolysis [103]. Numerous studies also demonstrated the overexpression of LDH-A in various types of cancer [104]. Considering the role of LDH-A in maintaining cancer cell energy metabolism, once its activity is inhibited, the energy-producing burden will be transferred to mitochondria, which may result in elevated oxidative stress and induce mitochondrial pathway apoptosis. Several studies have already found that the inhibition of LDH-A in cancer cells could stimulate mitochondrial respiration, decrease mitochondrial membrane potentials and finally lead to cancer cell death $[105,106]$. Given LDH-A inhibition has no significant toxic effect on normal tissue, it is promising to develop novel LDH-A inhibitors. Gossypol is a polyphenolic compound isolated from cotton seeds, which are traditionally used in TCM for improving immunity. Gossypol is initially applied as a male antifertility agent. Following studies suggest its anticancer, antioxidant, antiviral and antiparasitic activities [107-109]. Gossypol preferentially acts on redox reactions catalyzed by $\mathrm{NAD}^{+} / \mathrm{NADH}$-based enzymes such as LDH-A. Gossypol is a nonselective competitive LDH-A inhibitor and its anticancer activity appears to be associated with LDH-A inhibition [110, 111]. However, gossypol revealed significant toxicities including cardiac arrhythmias, renal failure, muscle weakness, and even paralysis, resulting in the stop of further development [112]. Galloflavin, a gallic acid derivative, was recently found to directly bind with LDH-A and LDH-B. Biological studies found that galloflavin could block aerobic glycolysis and trigger apoptosis in cancer cells without interfering cellular respiration. [113]. An antimalaria drug FX-11 was also reported to inhibit LDH-A activity and induce cancer growth arrest in both in vitro and 
in vivo experiments[114]. To explore active compounds from herbal medicine as LDH-A inhibitors, we investigated the effects of Spatholobus suberectus, a natural Chinese herb, on LDH-A activities in breast cancer cells. Our results showed that Spatholobus suberectus extractions significantly inhibited LDH-A activity in the breast cancer cells (unpublished data). Furthermore, we have conducted bioactivity-guided screening and epigallocatechin was identified as the main compound accounting for the herb anti-LDH-A function, the mechanism of which is correlated to accelerated HIF- $1 \alpha$ proteasome degradation (unpublished data).

3.4. Others. Glucose transporters (GLUTs) are important channels expressed on cell membrane for mediating glucose and other substrates entering into cells as nutrients. A total of six GLUT isoforms have been identified. Among them, GLUT-1 is closely related to cancer stages and chemo- or radiotherapy responses [115]. GLUT-1 silencing reduced cancer cell proliferation and mediated apoptosis [116]. For herb-derived inhibitors, apigenin, and genistein were proved to inhibit GLUT-1 [117, 118]. GAPDH is a classical glycolytic enzyme encoded by a "housekeeping gene" which is constitutively expressed in most cells. GAPDH is responsible for transforming glyceraldehydes-3-phosphate to 1,3-bisphosphoglycerate coupled with the reduction of $\mathrm{NAD}^{+}$to NADH. Beside glycolytic function, GAPDH also participated in endocytosis, membrane fusion, vesicular secretory, nuclear tRNA transport, and DNA replication or repair. GAPDH inhibition resulted in induction of apoptosis [119]. Arsenic was demonstrated to abolish ATP generation in GAPDH-catalysing reaction process, although it is not in a direct binding mode [120]. AMP-activated protein kinase (AMPK) serves as a critical sensor in monitoring intracellular energy supply [121]. AMPK contributes to the increase of glycolytic activity in cancer cells. Thus, AMPK becomes a novel therapeutic target for cancer treatment. Herb-derived compounds curcumin and quercetin were demonstrated to induce apoptosis via AMPK pathway in cancer cells $[122,123]$. In addition, other glycolytic enzymes including pyruvate kinase M2, glucose-6-phosphate isomerase, and transketolase-like enzyme 1, also participate in maintaining vitality of cancer cells. Development of small molecular inhibitors derived from herbs or natural plants targeting on these enzymes will be a new direction for anticancer research. The potential glycolysis inhibitors discussed above are summarized in the Figure 4.

In summary, recent research progress indicate that many active compounds derived from herbal medicine have the potentials to regulate key metabolic enzymes, such as HIF-1 $\alpha$, GLUT-1, hexokinase, LDH-A, and PDK1. Those enzymes and proteins are important signaling molecules in the glycolytic pathways of cancer cells. The unique glycolytic pathways could provide cancer cells sufficient energy and ATP for their rapid proliferation and growth. In the meantime, the unique metabolic characteristics of cancer cells raise great opportunities for the development of anticancer agents targeting on aerobic glycolysis. With this strategy, the compounds derived from herbal medicine or synthesized novel chemicals would preferentially kill cancer cells instead of normal cells by blocking aerobic glycolysis, greatly facilitating the drug discovery for molecular target therapy.

\section{Perspectives}

The elucidation of specific molecular signaling involved in cancer initiation, development, and metastasis have provided the grounds for molecular targeting based therapeutic strategy. Glycolysis is an important hallmark of cancer cells differentiated from normal cells. The metabolic alternations and adaptations of cancer cells have been extensively studied in last decades. Tumor cells exhibit altered metabolic behavior due to tumor cell intrinsic properties and tumor microenvironment. With Warburg effect, tumor cells have increased glucose uptake and preferentially metabolize glucose through glycolysis even in the presence of oxygen, allowing them to sustain higher proliferation rates and fast growth. Therefore, targeting on glycolysis can be an important strategy in cancer prevention and treatment. For example, cancer cells acquire and develop resistance in many patients when receiving chemotherapy or radiotherapy. A recent study investigated the antitumor effects of trastuzumab (a monoclonal antibody against EGFR-2) in combination with glycolysis inhibitor 2-DG in ErbB2positive breast cancer. Trastuzumab inhibited glycolysis via downregulation of heat shock factor 1 (HSF1) and LDHA in ErbB2-positive cancer cells, resulting in tumor growth inhibition. Moreover, increased glycolysis via HSF1 and LDH-A contributed to trastuzumab resistance. Combining trastuzumab with glycolysis inhibition synergistically inhibited trastuzumab-sensitive and -resistant breast cancers in vitro and in vivo, due to more efficient inhibition of glycolysis [124]. Thus, inhibition of glycolysis may offer a promising strategy to overcome the resistances of cancer cells toward chemotherapy $[125,126]$. Similarly, glycolysis based on Warburg effect also links to radioresistance [127]. Given many herb-derived phytochemicals exhibit properties of antiglycolysis and reversal of drug-resistance, Chinese herbal medicine targeting cancer glycolysis can be developed as an adjunct treatment for cancer patients by combining chemotherapy and radiotherapy. It will provide an opportunity to increase clinical outcome in cancer treatment.

There are 250,000 to 300,000 plant species in the world. Although large efforts are made, only 5,000 plant species have been studied for their possible medical applications. With the long history of application in human subjects, Chinese herbal medicine enjoys a unique position for molecular targeting-based therapeutic strategy. Based on histological documents and case reports in cancer treatment, Chinese herbal medicine provides a fast track and important source in drug discovery for molecular targeting based therapeutic strategy. It is anticipated that in the years to come, more and more medicinal herbs will be screened targeting on glycolytic-related molecular targets or other therapeutic targets. Establishing well validated high throughput screening platform is necessary and essential for the purpose, greatly accelerating the process of drug development. In conclusion, molecular-targeted screening strategy is critical and efficient 
<smiles>O=C(CC(=O)c1c(O)cc(O)cc1O)c1ccc(O)cc1</smiles>

(Apigenin)<smiles>COc1cc(/C=C/C(=O)/C=C(O)/C=C/c2ccc(O)c(OC)c2)ccc1O</smiles>

(Curcumin)

(a)<smiles>OCC1O[C@H](O)C[C@@H](O)[C@H]1O</smiles>

(2-deoxy-glucose)<smiles>Cc1ccc(C(C)C)c(O)c1C=O</smiles>

(Gossypol)<smiles>O=C(O)C(=O)CBr</smiles>

(3-bromo-pyruvate)

(b)<smiles>O=c1oc2c(cc1O)oc(=O)c1cc(O)c(O)c(O)c12</smiles>

(Galloflavin)<smiles>O=C(O[C@H]1Cc2c(O)cc(O)cc2O[C@@H]1c1cc(O)c(O)c(O)c1)c1cc(O)c(O)c(O)c1</smiles>

(Epigallocatechin gallate)<smiles>CC[C@@]1(O)C(=O)OCc2c1cc1n(c2=O)Cc2cc3ccccc3nc2-1</smiles>

(Camptothecin)

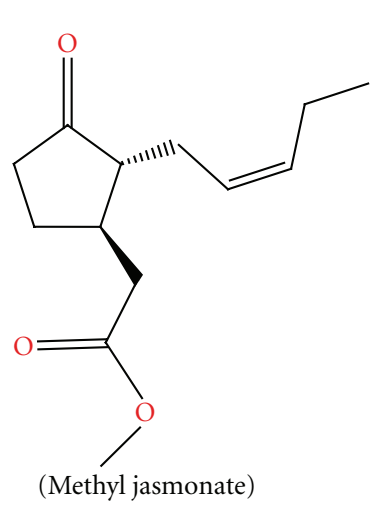<smiles>CCCc1c(O)c(O)c(C(=O)O)c2cc(Cc3ccccc3)c(C)cc12</smiles>

(FX-11)

(c)

FIGURE 4: Chemical structures of glycolytic inhibitors derived from Chinese herbs. (a) Chemicals targeting on HIF-1 $\alpha$; (b) HKII inhibitors; (c) Chemicals targeting on LDH-A. 
strategy for exploring the active compounds from Chinese herbal medicine for anticancer drug discovery. It will not only bring the discoveries of new anticancer drugs with more target specific and low toxic, but also make contributions to the globalization and modernization of traditional Chinese medicine.

\section{Abbreviations}

TCM: Traditional Chinese medicine

CAM: Complementary and alternative medicine

RCT: Randomized controlled trials

GLUT-1: Glucose transporter-1

SGLT-1: Sodium glucose cotransporter-1

PGK: Phosphoglycerate kinase

PK: $\quad$ Pyruvate kinase

LDH: Lactate dehydrogenase

MCT: Monocarboxylate transporter

GAPDH: Glyceraldehydes-3-phosphate dehydrogenase

PKM2: $\quad$ Pyruvate kinase, muscle

HIF-1: Hypoxia inducible factor-1

ODD: Oxygen-dependent degradation domain

VHL: von Hippel-Lindau

EGCG: Epigallacatechin gallate

HK: Hexokinase

VDAC: Voltage-dependent anion channel

2-DG: 2-Deoxy-glucose

3-BrpA: 3-Bromo-pyruvate

AMPK: AMP-activated protein kinase

HSF1: Heat shock factor 1.

\section{Conflict of Interests}

The authors declare that there are no conflict of interests.

\section{Acknowledgments}

This work was supported by the Hong Kong Innovation and Technology Support Programme ITF funding (ITS/073/11FP), by the Seed Funding Programme for Applied Research, University of Hong Kong (201109160022), by the and Seed Funding Programme for Basic Research, University of Hong Kong (201011159053).

\section{References}

[1] A. Jemal, R. Siegel, J. Xu, and E. Ward, "Cancer statistics, 2010," CA Cancer Journal for Clinicians, vol. 60, no. 5, pp. 277-300, 2010.

[2] A. Ocana, A. Pandiella, L. L. Siu, and I. F. Tannock, "Preclinical development of molecular-targeted agents for cancer," Nature Reviews Clinical Oncology, vol. 8, no. 4, pp. 200-209, 2011.

[3] P. Sharma, K. Wagner, J. D. Wolchok, and J. P. Allison, "Novel cancer immunotherapy agents with survival benefit: recent successes and next steps," Nature Reviews Cancer, vol. 11, no. 11 , pp. 805-812, 2011.

[4] A. Prat and J. Baselga, "The role of hormonal therapy in the management of hormonal-receptor-positive breast cancer with co-expression of HER2," Nature Clinical Practice Oncology, vol. 5, no. 9, pp. 531-542, 2008.

[5] E. Ernst, "Complementary and alternative medicine (CAM) and cancer: the kind face of complementary medicine," International Journal of Surgery, vol. 7, no. 6, pp. 499-500, 2009.

[6] S. Miller, J. Stagl, D. B. Wallerstedt, M. Ryan, and P. J. Mansky, "Botanicals used in complementary and alternative medicine treatment of cancer: clinical science and future perspectives," Expert Opinion on Investigational Drugs, vol. 17, no. 9, pp. 1353-1364, 2008.

[7] B. Carmady and C. A. Smith, "Use of Chinese medicine by cancer patients: a review of surveys," Chinese Medicine, vol. 6, p. 22, 2011.

[8] W. L. W. Hsiao and L. Liu, "The role of traditional Chinese herbal medicines in cancer therapy-from TCM theory to mechanistic insights," Planta Medica, vol. 76, no. 11, pp. 1118-1131, 2010.

[9] H. Ouyang, P. Wang, Z. Meng et al., "Multimodality treatment of pancreatic cancer with liver metastases using chemotherapy, radiation therapy, and/or Chinese herbal medicine," Pancreas, vol. 40, no. 1, pp. 120-125, 2011.

[10] S. Wang, S. Penchala, S. Prabhu, J. Wang, and Y. Huang, "Molecular basis of traditional chinese medicine in cancer chemoprevention," Current Drug Discovery Technologies, vol. 7, no. 1, pp. 67-75, 2010.

[11] H. S. Parekh, G. Liu, and M. Q. Wei, "A new dawn for the use of traditional Chinese medicine in cancer therapy," Molecular Cancer, vol. 8, p. 21, 2009.

[12] W. J. Craig, "Phytochemicals: guardians of our health," Journal of the American Dietetic Association, vol. 97, no. 10, pp. S199-S204, 1997.

[13] W. J. Craig, "Health-promoting properties of common herbs," The American Journal of Clinical Nutrition, vol. 70, no. 3, pp. 491S-499S, 1999.

[14] D. J. Newman, G. M. Cragg, and K. M. Snader, "Natural products as sources of new drugs over the period 1981-2002," Journal of Natural Products, vol. 66, no. 7, pp. 1022-1037, 2003.

[15] T. Efferth, P. C. H. Li, V. S. B. Konkimalla, and B. Kaina, "From traditional Chinese medicine to rational cancer therapy," Trends in Molecular Medicine, vol. 13, no. 8, pp. 353-361, 2007.

[16] C. W. Cheng, W. Fan, S. G. Ko, L. Song, and Z. X. Bian, "Evidence-based management of herb-drug interaction in cancer chemotherapy," Explore, vol. 6, no. 5, pp. 324-329, 2010.

[17] S. Wang, S. Penchala, S. Prabhu, J. Wang, and Y. Huang, "Molecular basis of traditional chinese medicine in cancer chemoprevention," Current Drug Discovery Technologies, vol. 7, no. 1, pp. 67-75, 2010.

[18] B. B. Aggarwal, H. Ichikawa, P. Garodia et al., "From traditional Ayurvedic medicine to modern medicine: identification of therapeutic targets for suppression of inflammation and cancer," Expert Opinion on Therapeutic Targets, vol. 10, no. 1, pp. 87-118, 2006.

[19] K. L. Yim and D. Cunningham, "Targeted drug therapies and cancer," Recent Results in Cancer Research, vol. 185, pp. 159$171,2011$.

[20] K. Takeuchi and F. Ito, "Receptor tyrosine kinases and targeted cancer therapeutics," Biologilca and Pharmaceutical Bulletin, vol. 34, no. 12, pp. 1774-1780, 2011. 
[21] H. Allgayer and S. Fulda, "An introduction to molecular targeted therapy of cancer," Advances in Medical Sciences, vol. 53, no. 2, pp. 130-138, 2008.

[22] E. Nadal and E. Olavarria, "Imatinib mesylate (Gleevec/ Glivec) a molecular-targeted therapy for chronic myeloid leukaemia and other malignancies," International Journal of Clinical Practice, vol. 58, no. 5, pp. 511-516, 2004.

[23] R. Nahta and F. J. Esteva, "HER-2-targeted therapy: lessons learned and future directions," Clinical Cancer Research, vol. 9, no. 14, pp. 5078-5084, 2003.

[24] G. Vlacich and R. Coffey, "Resistance to EGFR-targeted therapy: a family affair," Cancer Cell, vol. 20, no. 4, pp. 423-425, 2011.

[25] B. I. Rini and M. B. Atkins, "Resistance to targeted therapy in renal-cell carcinoma," The Lancet Oncology, vol. 10, no. 10, pp. 992-1000, 2009.

[26] M. G. Vander Heiden, "Targeting cancer metabolism: a therapeutic window opens," Nature Reviews Drug Discovery, vol. 10, no. 9, pp. 671-684, 2011.

[27] L. M. R. Ferreira, "Cancer metabolism: the Warburg effect today," Experimental and Molecular Pathology, vol. 89, no. 3, pp. 372-380, 2010.

[28] A. Najafov and D. R. Alessi, "Uncoupling the warburg effect from cancer," Proceedings of the National Academy of Sciences of the United States of America, vol. 107, no. 45, pp. 1913519136, 2010.

[29] X. Liu, X. Wang, J. Zhang et al., "Warburg effect revisited: an epigenetic link between glycolysis and gastric carcinogenesis," Oncogene, vol. 29, no. 3, pp. 442-450, 2010.

[30] H. Lu, R. A. Forbes, and A. Verma, "Hypoxia-inducible factor 1 activation by aerobic glycolysis implicates the Warburg effect in carcinogenesis," Journal of Biological Chemistry, vol. 277, no. 26, pp. 23111-23115, 2002.

[31] H. Pelicano, D. S. Martin, R. H. Xu, and P. Huang, "Glycolysis inhibition for anticancer treatment," Oncogene, vol. 25, no. 34, pp. 4633-4646, 2006.

[32] P. E. Porporato, S. Dhup, R. K. Dadhich, T. Copetti, and P. Sonveaux, "Anticancer targets in the glycolytic metabolism of tumors: a comprehensive review," Frontiers in Pharmacology, vol. 2, p. 49, 2011.

[33] O. Warburg, "On respiratory impairment in cancer cells," Science, vol. 124, no. 3215, pp. 269-270, 1956.

[34] L. M. R. Ferreira, "Cancer metabolism: the Warburg effect today," Experimental and Molecular Pathology, vol. 89, no. 3, pp. 372-380, 2010.

[35] P. P. Hsu and D. M. Sabatini, "Cancer cell metabolism: Warburg and beyond," Cell, vol. 134, no. 5, pp. 703-707, 2008.

[36] K. Garber, "Energy boost: the Warburg effect returns in a new theory of cancer," Journal of the National Cancer Institute, vol. 96, no. 24, pp. 1805-1806, 2004.

[37] J. P. Bayley and P. Devilee, "The Warburg effect in 2012," Current Opinion in Oncology, vol. 24, no. 1, pp. 62-67, 2012.

[38] M. Stubbs and J. R. Griffiths, "The altered metabolism of tumors: HIF-1 and its role in the Warburg effect," Advances in Enzyme Regulation, vol. 50, no. 1, pp. 44-55, 2010.

[39] M. G. Vander Heiden, L. C. Cantley, and C. B. Thompson, "Understanding the Warburg effect: the metabolic requirements of cell proliferation," Science, vol. 324, no. 5930, pp. 1029-1033, 2009.

[40] M. Shanmugam, S. K. McBrayer, and S. T. Rosen, "Targeting the Warburg effect in hematological malignancies: from PET to therapy," Current Opinion in Oncology, vol. 21, no. 6, pp. 531-536, 2009.

[41] Z. Chen, W. Lu, C. Garcia-Prieto, and P. Huang, "The Warburg effect and its cancer therapeutic implications," Journal of Bioenergetics and Biomembranes, vol. 39, no. 3, pp. 267-274, 2007.

[42] R. Scatena, P. Bottoni, A. Pontoglio, and B. Giardina, "Revisiting the Warburg effect in cancer cells with proteomics. The emergence of new approaches to diagnosis, prognosis and therapy," Proteomics Clinical Applications, vol. 4, no. 2, pp. 143-158, 2010.

[43] R. A. Gatenby and R. J. Gillies, "Glycolysis in cancer: a potential target for therapy," International Journal of Biochemistry \& Cell Biology, vol. 39, no. 7-8, pp. 1358-1366, 2007.

[44] S. Y. Lunt and M. G. Vander Heiden, "Aerobic glycolysis: meeting the metabolic requirements of cell proliferation," Annual Review of Cell and Developmental Biology, vol. 27, pp. 441-464, 2011.

[45] K. Podar and K. C. Anderson, "A therapeutic role for targeting c-Myc/Hif-1-dependent signaling pathways," Cell Cycle, vol. 9, no. 9, pp. 1722-1728, 2010.

[46] Y. G. Yoo, M. Hayashi, J. Christensen, and L. E. Huang, "An essential role of the HIF- $1 \alpha$-c-Myc axis in malignant progression," Annals of the New York Academy of Sciences, vol. 1177, pp. 198-204, 2009.

[47] R. S. Brown, T. M. Goodman, K. R. Zasadny, J. K. Greenson, and R. L. Wahl, "Expression of hexokinase II and Glut-1 in untreated human breast cancer," Nuclear Medicine and Biology, vol. 29, no. 4, pp. 443-453, 2002.

[48] Y. Kolev, H. Uetake, Y. Takagi, and K. Sugihara, "Lactate dehydrogenase-5 (LDH-5) expression in human gastric cancer: association with hypoxia-inducible factor (HIF-1 $\alpha$ ) pathway, angiogenic factors production and poor prognosis," Annals of Surgical Oncology, vol. 15, no. 8, pp. 2336-2344, 2008.

[49] Y. Higashimura, Y. Nakajima, R. Yamaji et al., "Up-regulation of glyceraldehyde-3-phosphate dehydrogenase gene expression by HIF-1 activity depending on Sp1 in hypoxic breast cancer cells," Archives of Biochemistry and Biophysics, vol. 509, no. 1, pp. 1-8, 2011.

[50] H. Xie, V. A. Valera, M. J. Merino et al., "LDH-A inhibition, a therapeutic strategy for treatment of hereditary leiomyomatosis and renal cell cancer," Molecular Cancer Therapeutics, vol. 8, no. 3, pp. 626-635, 2009.

[51] H. S. Shi, D. Li, J. Zhang et al., "Silencing of $p k m 2$ increases the efficacy of docetaxel in human lung cancer xenografts in mice," Cancer Science, vol. 101, no. 6, pp. 1447-1453, 2010.

[52] M. Zhou, Y. Zhao, Y. Ding et al., "Warburg effect in chemosensitivity: targeting lactate dehydrogenase-A resensitizes Taxol-resistant cancer cells to Taxol," Molecular Cancer, vol. 9, p. 33, 2010.

[53] T. Ogino, T. Yamadera, T. Nonaka, S. Imajoh-Ohmi, and K. Mizumoto, "Enolase, a cellular glycolytic enzyme, is required for efficient transcription of Sendai virus genome," Biochemical and Biophysical Research Communications, vol. 285, no. 2, pp. 447-455, 2001.

[54] J. G. Pastorino and J. B. Hoek, "Hexokinase II: the integration of energy metabolism and control of apoptosis," Current Medicinal Chemistry, vol. 10, no. 16, pp. 1535-1551, 2003.

[55] Y. Dobashi, H. Watanabe, M. Matsubara et al., "Autocrine motility factor/glucose-6-phosphate isomerase is a possible predictor of metastasis in bone and soft tissue tumours," Journal of Pathology, vol. 208, no. 1, pp. 44-53, 2006. 
[56] G. L. Semenza, "HIF-1: upstream and downstream of cancer metabolism," Current Opinion in Genetics \& Development, vol. 20, no. 1, pp. 51-56, 2010.

[57] M. C. Lauzier, M. D. Michaud, M. A. C. Déry, and D. E. Richard, "HIF-1 activation during tumor progression: implications and consequences," Bulletin du Cancer, vol. 93, no. 4, pp. 349-356, 2006.

[58] E. Monti and M. B. Gariboldi, "HIF-1 as a target for cancer chemotherapy, chemosensitization and chemoprevention," Current Molecular Pharmacology, vol. 4, no. 1, pp. 62-77, 2011.

[59] J. M. Adams, L. T. Difazio, R. H. Rolandelli et al., "HIF-1: a key mediator in hypoxia (Review)," Acta Physiologica Hungarica, vol. 96, no. 1, pp. 19-28, 2009.

[60] G. L. Semenza, "Hypoxia-inducible factor 1 (HIF-1) pathway," Science's STKE, vol. 407, p. cm8, 2007.

[61] M. Y. Koh, T. R. Spivak-Kroizman, and G. Powis, "HIF-1 regulation: not so easy come, easy go," Trends in Biochemical Sciences, vol. 33, no. 11, pp. 526-534, 2008.

[62] J. E. Ziello, I. S. Jovin, and Y. Huang, "Hypoxia-Inducible Factor (HIF)-1 regulatory pathway and its potential for therapeutic intervention in malignancy and ischemia," Yale Journal of Biology and Medicine, vol. 80, no. 2, pp. 51-60, 2007.

[63] G. L. Semenza, "Targeting HIF-1 for cancer therapy," Nature Reviews Cancer, vol. 3, no. 10, pp. 721-732, 2003.

[64] G. L. Semenza, "HIF-1 inhibitors for cancer therapy: from gene expression to drug discovery," Current Pharmaceutical Design, vol. 15, no. 33, pp. 3839-3843, 2009.

[65] E. J. Choi and G. H. Kim, "Apigenin induces apoptosis through a mitochondria/caspase-pathway in human breast cancer MDA-MB-453 cells," Journal of Clinical Biochemistry and Nutrition, vol. 44, no. 3, pp. 260-265, 2009.

[66] Z. D. Li, X. W. Hu, Y. T. Wang, and J. Fang, "Apigenin inhibits proliferation of ovarian cancer A2780 cells through Id1," FEBS Letters, vol. 583, no. 12, pp. 1999-2003, 2009.

[67] S. Shukla and S. Gupta, "Molecular targets for apigenininduced cell cycle arrest and apoptosis in prostate cancer cell xenograft," Molecular Cancer Therapeutics, vol. 5, no. 4, pp. 843-852, 2006.

[68] H. F. Lu, Y. U. J. Chie, M. S. Yang et al., "Apigenin induces caspase-dependent apoptosis in human lung cancer A549 cells through Bax- and Bcl-2-triggered mitochondrial pathway," International Journal of Oncology, vol. 36, no. 6, pp. 1477-1484, 2010.

[69] J. Fang, Q. Zhou, L. Z. Liu et al., "Apigenin inhibits tumor angiogenesis through decreasing HIF- $1 \alpha$ and VEGF expression," Carcinogenesis, vol. 28, no. 4, pp. 858-864, 2007.

[70] L. Z. Liu, J. Fang, Q. Zhou, X. Hu, X. Shi, and B. H. Jiang, "Apigenin inhibits expression of vascular endothelial growth factor and angiogenesis in human lung cancer cells: implication of chemoprevention of lung cancer," Molecular Pharmacology, vol. 68, no. 3, pp. 635-643, 2005.

[71] S. Shukla and S. Gupta, "Apigenin-induced cell cycle arrest is mediated by modulation of MAPK, PI3K-Akt, and loss of cyclin D1 associated retinoblastoma dephosphorylation in human prostate cancer cells," Cell Cycle, vol. 6, no. 9, pp. 1102-1114, 2007.

[72] P. Kaur, S. Shukla, and S. Gupta, "Plant flavonoid apigenin inactivates Akt to trigger apoptosis in human prostate cancer: an in vitro and in vivo study," Carcinogenesis, vol. 29, no. 11, pp. 2210-2217, 2008.
[73] S. Mirzoeva, N. D. Kim, K. Chiu, C. A. Franzen, R. C. Bergan, and J. C. Pelling, "Inhibition of HIF-1 alpha and VEGF expression by the chemopreventive bioflavonoid apigenin is accompanied by Akt inhibition in human prostate carcinoma PC3-M cells," Molecular Carcinogenesis, vol. 47, no. 9, pp. 686-700, 2008.

[74] M. Osada, S. Imaoka, and Y. Funae, "Apigenin suppresses the expression of VEGF, an important factor for angiogenesis, in endothelial cells via degradation of HIF- $1 \alpha$ protein," FEBS Letters, vol. 575, no. 1-3, pp. 59-63, 2004.

[75] S. Samarghandian, J. T. Afshari, and S. Davoodi, "Chrysin reduces proliferation and induces apoptosis in the human prostate cancer cell line pc-3," Clinics, vol. 66, no. 6, pp. 10731079, 2011.

[76] T. Phan, X. M. Yu, M. Kunnimalaiyaan, and H. Chen, "Antiproliferative effect of chrysin on anaplastic thyroid cancer," Journal of Surgical Research, vol. 170, no. 1, pp. 8488, 2011.

[77] B. Y. Khoo, S. L. Chua, and P. Balaram, "Apoptotic effects of chrysin in human cancer cell lines," International Journal of Molecular Sciences, vol. 11, no. 5, pp. 2188-2199, 2010.

[78] B. Fu, J. Xue, Z. Li, X. Shi, B. H. Jiang, and J. Fang, "Chrysin inhibits expression of hypoxia-inducible factor $1-\alpha$ through reducing hypoxia-inducible factor- $1 \alpha$ stability and inhibiting its protein synthesis," Molecular Cancer Therapeutics, vol. 6, no. 1, pp. 220-226, 2007.

[79] B. N. Singh, S. Shankar, and R. K. Srivastava, "Green tea catechin, epigallocatechin-3-gallate (EGCG): mechanisms, perspectives and clinical applications," Pharmacology, vol. 82, no. 12, pp. 1807-1821, 2011.

[80] D. G. Nagle, D. Ferreira, and Y. D. Zhou, "Epigallocatechin3-gallate (EGCG): chemical and biomedical perspectives," Phytochemistry, vol. 67, no. 17, pp. 1849-1855, 2006.

[81] N. Khan, F. Afaq, M. Saleem, N. Ahmad, and H. Mukhtar, "Targeting multiple signaling pathways by green tea polyphenol (-)-epigallocatechin-3-gallate," Cancer Research, vol. 66, no. 5, pp. 2500-2505, 2006.

[82] H. Tachibana, "Molecular basis for cancer chemoprevention by green tea polyphenol EGCG," Forum of Nutrition, vol. 61, pp. 156-169, 2009.

[83] Q. Zhang, X. Tang, Q. Lu, Z. Zhang, J. Rao, and A. D. Le, "Green tea extract and (-)-epigallocatechin-3-gallate inhibit hypoxia- and serum-induced HIF-1protein accumulation and VEGF expression in human cervical carcinoma and hepatoma cells," Molecular Cancer Therapeutics, vol. 5, no. 5, pp. 1227-1238, 2006.

[84] H. Zhou, C. S. Beevers, and S. Huang, "The targets of curcumin," Current Drug Targets, vol. 12, no. 3, pp. 332-347, 2011.

[85] D. M. Hossain, S. Bhattacharyya, T. Das, and G. Sa, "Curcumin: the multi-targeted therapy for cancer regression," Frontiers in Biosciences, vol. 4, pp. 335-355, 2012.

[86] M. K. Bae, S. H. Kim, J. W. Jeong et al., "Curcumin inhibits hypoxia-induced angiogenesis via down-regulation of HIF1," Oncology Reports, vol. 15, no. 6, pp. 1557-1562, 2006.

[87] M. Ströfer, W. Jelkmann, and R. Depping, "Curcumin decreases survival of Hep3B liver and MCF-7 Breast Cancer Cells-the role of HIF," Strahlentherapie und Onkologie, vol. 187, no. 7, pp. 393-400, 2011.

[88] P. Klausmeyer, T. C. McCloud, G. Melillo, D. A. Scudiero, J. H. Cardellina, and R. H. Shoemaker, "Identification of a new natural camptothecin analogue in targeted screening for 
HIF-1 $\alpha$ inhibitors," Planta Medica, vol. 73, no. 1, pp. 49-52, 2007.

[89] T. D. Nguyen, X. Jin, J. H. Lee et al., "Abietane diterpenes from Salvia miltiorrhiza inhibit the activation of hypoxiainducible factor-1," Journal of Natural Products, vol. 70, no. 7, pp. 1093-1097, 2007.

[90] S. Kawai, T. Mukai, S. Mori, B. Mikami, and K. Murata, "Hypothesis: structures, evolution, and ancestor of glucose kinases in the hexokinase family," Journal of Bioscience and Bioengineering, vol. 99, no. 4, pp. 320-330, 2005.

[91] Y. Fan and W. X. Zong, "Hacking hexokinase halts tumor growth," Cancer Biology and Therapy, vol. 7, no. 7, pp. 1136$1138,2008$.

[92] A. Rempel, S. P. Mathupala, C. A. Griffin, A. L. Hawkins, and P. L. Pedersen, "Glucose catabolism in cancer cells: amplification of the gene encoding type II hexokinase," Cancer Research, vol. 56, no. 11, pp. 2468-2471, 1996.

[93] J. G. Pastorino and J. B. Hoek, "Regulation of hexokinase binding to VDAC," Journal of Bioenergetics and Biomembranes, vol. 40, no. 3, pp. 171-182, 2008.

[94] L. Galluzzi, O. Kepp, N. Tajeddine, and G. Kroemer, "Disruption of the hexokinase-VDAC complex for tumor therapy," Oncogene, vol. 27, no. 34, pp. 4633-4635, 2008.

[95] R. L. Aft, F. W. Zhang, and D. Gius, "Evaluation of 2-deoxyD-glucose as a chemotherapeutic agent: mechanism of cell death," British Journal of Cancer, vol. 87, no. 7, pp. 805-812, 2002.

[96] P. Dell'Antone, "Targets of 3-bromopyruvate, a new, energy depleting, anticancer agent," Medicinal Chemistry, vol. 5, no. 6, pp. 491-496, 2009.

[97] H. L. Yang, H. X. Zhang, L. H. Li et al., "Effect of bencao xiaoke pill on carbohydrate metabolism enzyme activity in type 2 diabetic rats," Lishizhen Medicine and Materia Medica Research, vol. 20, no. 7, pp. 1615-1616, 2009.

[98] D. E. Ezekwudo, R. C. Wang, and J. A. Elegbede, "Methyl jasmonate induced apoptosis in human prostate carcinoma cells via 5-lipoxygenase dependent pathway," Journal of Experimental Therapeutics \& Oncology, vol. 6, no. 4, pp. 267277, 2007.

[99] E. Milrot, A. Jackman, T. Kniazhanski, P. Gonen, E. Flescher, and L. Sherman, "Methyl jasmonate reduces the survival of cervical cancer cells and downregulates HPV E6 and E7, and surviving," Cancer Letters, vol. 319, no. 1, pp. 31-38, 2012.

[100] X. Y. Xiao, G. S. Jiang, L. Wang, L. Lv, and F. Q. Zeng, "Predominant enhancement of apoptosis induced by methyl jasmonate in bladder cancer cells: therapeutic effect of the antp-conjugated Smac peptide," Anti-Cancer Drugs, vol. 22, no. 9, pp. 853-863, 2011.

[101] N. Goldin, L. Arzoine, A. Heyfets et al., "Methyl jasmonate binds to and detaches mitochondria-bound hexokinase," Oncogene, vol. 27, no. 34, pp. 4636-4643, 2008.

[102] K. Augoff and K. Grabowski, "Significance of lactate dehydrogenase measurements in diagnosis of malignancies," Polski Merkuriusz Lekarski, vol. 17, no. 102, pp. 644-647, 2004.

[103] T. Kanno, K. Sudo, and I. Takeuchi, "Hereditary deficiency of lactate dehydrogenase M-subunit," Clinica Chimica Acta, vol. 108, no. 2, pp. 267-276, 1980.

[104] M. I. Koukourakis, A. Giatromanolaki, S. Winter, R. Leek, E. Sivridis, and A. L. Harris, "Lactate dehydrogenase 5 expression in squamous cell head and neck cancer relates to prognosis following radical or postoperative radiotherapy," Oncology, vol. 77, no. 5, pp. 285-292, 2009.
[105] A. Le, C. R. Cooper, A. M. Gouw et al., "Inhibition of lactate dehydrogenase A induces oxidative stress and inhibits tumor progression," Proceedings of the National Academy of Sciences of the United States of America, vol. 107, no. 5, pp. 2037-2042, 2010.

[106] Z. Y. Wang, T. Y. Loo, J. G. Shen et al., "LDH-A silencing suppresses breast cancer tumorigenicity through induction of oxidative stress mediated mitochondrial pathway apoptosis," Breast Cancer Research and Treatment, vol. 131, no. 3, pp. 791-800, 2012.

[107] X. Pang, Y. Wu, Y. Wu et al., “(-)-Gossypol suppresses the growth of human prostate cancer xenografts via modulating VEGF signaling-mediated angiogenesis," Molecular Cancer Therapeutics, vol. 10, no. 5, pp. 795-805, 2011.

[108] M. J. Sikora, J. A. Bauer, M. Verhaegen et al., "Antioxidant treatment enhances anti-tumor cytotoxicity of (-)gossypol," Cancer Biology \& Therapy, vol. 7, no. 5, pp. 767776, 2008.

[109] D. L. Vander Jagt, L. M. Deck, and R. E. Royer, "Gossypol: prototype of inhibitors targeted to dinucleotide folds," Current Medicinal Chemistry, vol. 7, no. 4, pp. 479-498, 2000.

[110] R. Conners, F. Schambach, J. Read et al., "Mapping the binding site for gossypol-like inhibitors of Plasmodium falciparum lactate dehydrogenase," Molecular and Biochemical Parasitology, vol. 142, no. 2, pp. 137-148, 2005.

[111] M. H. Javed and M. A. Khan, "Effect of amino acids on inhibition of lactate dehydogenase-X by gossypol," Experimental and Molecular Medicine, vol. 31, no. 1, pp. 25-29, 1999.

[112] P. Kovaci, "Mechanism of drug and toxic actions of gossypol: focus on reactive oxygen species and electron transfer," Current Medicinal Chemistry, vol. 10, no. 24, pp. 2711-2718, 2003.

[113] M. Manerba, M. Vettraino, L. Fiume et al., "Galloflavin (CAS 568-80-9): a novel inhibitor of lactate dehydrogenase," ChemMedChem, vol. 7, no. 2, pp. 311-317, 2012.

[114] A. Le, C. R. Cooper, A. M. Gouw et al., "Inhibition of lactate dehydrogenase A induces oxidative stress and inhibits tumor progression," Proceedings of the National Academy of Sciences of the United States of America, vol. 107, no. 5, pp. 2037-2042, 2010.

[115] S. Brophy, K. M. Sheehan, D. A. McNamara, J. Deasy, D. J. Bouchier-Hayes, and E. W. Kay, "GLUT-1 expression and response to chemoradiotherapy in rectal cancer," International Journal of Cancer, vol. 125, no. 12, pp. 2778-2782, 2009.

[116] S. Rastogi, S. Banerjee, S. Chellappan, and G. R. Simon, "Glut-1 antibodies induce growth arrest and apoptosis in human cancer cell lines," Cancer Letters, vol. 257, no. 2, pp. 244-251, 2007.

[117] L. G. Melstrom, M. R. Salabat, X. Z. Ding et al., "Apigenin inhibits the GLUT-1 glucose transporter and the phosphoinositide 3-kinase/akt pathway in human pancreatic cancer cells," Pancreas, vol. 37, no. 4, pp. 426-431, 2008.

[118] J. C. Vera, A. M. Reyes, J. G. Cárcamo et al., "Genistein is a natural inhibitor of hexose and dehydroascorbic acid transport through the glucose transporter, GLUT1," Journal of Biological Chemistry, vol. 271, no. 15, pp. 8719-8724, 1996.

[119] A. Tarze, A. Deniaud, M. le Bras et al., "GAPDH, a novel regulator of the pro-apoptotic mitochondrial membrane permeabilization," Oncogene, vol. 26, no. 18, pp. 2606-2620, 2007.

[120] Z. Gregus and B. Németi, "The glycolytic enzyme glyceraldehyde-3-phosphate dehydrogenase works as an arsenate 
reductase in human red blood cells and rat liver cytosol," Toxicological Sciences, vol. 85, no. 2, pp. 859-869, 2005.

[121] Z. Luo, M. Zang, and W. Guo, "AMPK as a metabolic tumor suppressor: control of metabolism and cell growth," Future Oncology, vol. 6, no. 3, pp. 457-470, 2010.

[122] W. Pan, H. Yang, C. Cao et al., "AMPK mediates curcumininduced cell death in CaOV3 ovarian cancer cells," Oncology Reports, vol. 20, no. 6, pp. 1553-1559, 2008.

[123] H. J. Kim, S. K. Kim, B. S. Kim et al., "Apoptotic effect of quercetin on HT-29 colon cancer cells via the AMPK signaling pathway," Journal of Agricultural and Food Chemistry, vol. 58, no. 15, pp. 8643-8650, 2010.

[124] Y. Zhao, H. Liu, Z. Liu et al., "Overcoming trastuzumab resistance in breast cancer by targeting dysregulated glucose metabolism," Cancer Research, vol. 71, no. 13, pp. 4585-4597, 2011.

[125] D. H. Suh, M. K. Kim, J. H. No, H. H. Chung, and Y. S. Song, "Metabolic approaches to overcoming chemoresistance in ovarian cancer," Annals of the New York Academy of Sciences, vol. 1229, no. 1, pp. 53-60, 2011.

[126] M. Zhou, Y. Zhao, Y. Ding et al., "Warburg effect in chemosensitivity: targeting lactate dehydrogenase-A re-sensitizes taxol-resistant cancer cells to taxol," Molecular Cancer, vol. 9, p. 33, 2010.

[127] S. P. Pitroda, B. T. Wakim, R. F. Sood et al., "STAT1dependent expression of energy metabolic pathways links tumour growth and radioresistance to the Warburg effect," BMC Medicine, vol. 7, p. 68, 2009. 


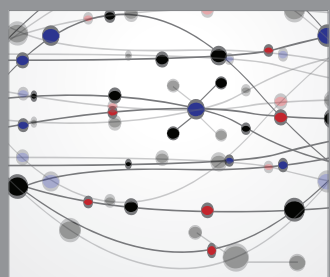

The Scientific World Journal
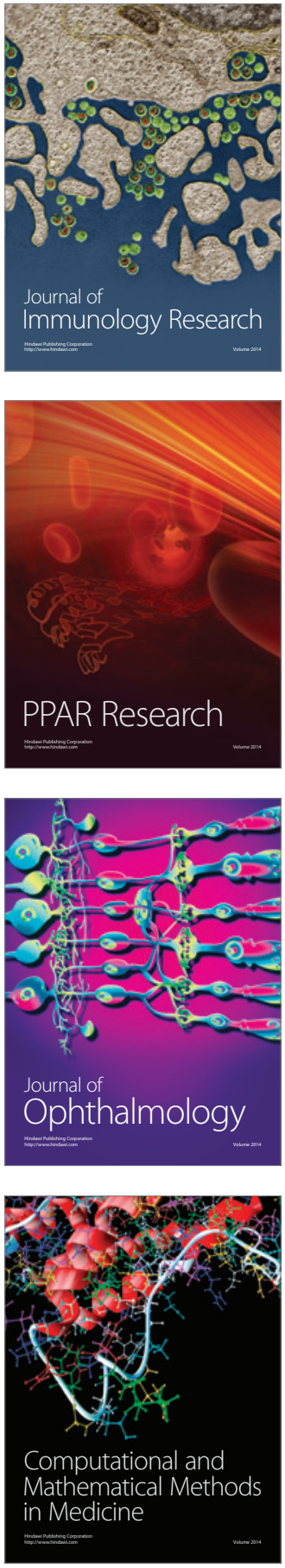

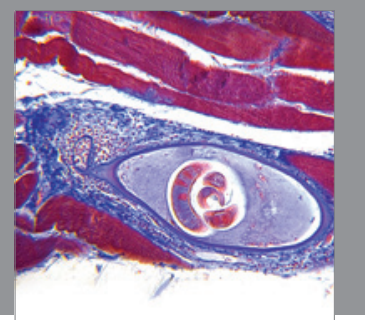

Gastroenterology

Research and Practice
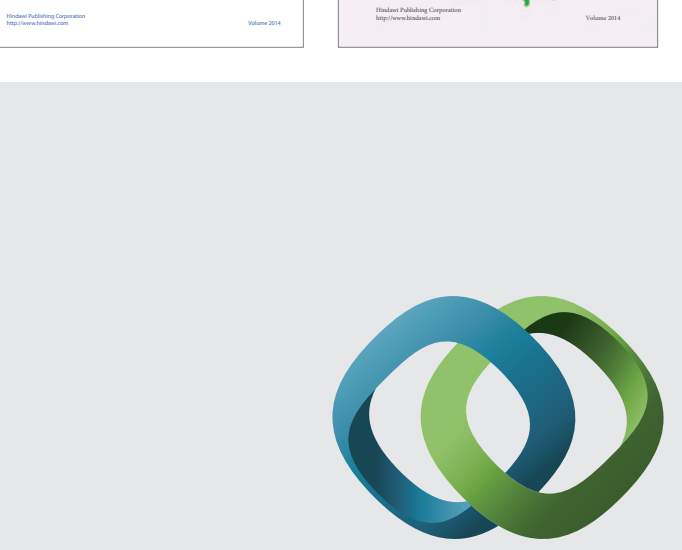

\section{Hindawi}

Submit your manuscripts at

http://www.hindawi.com
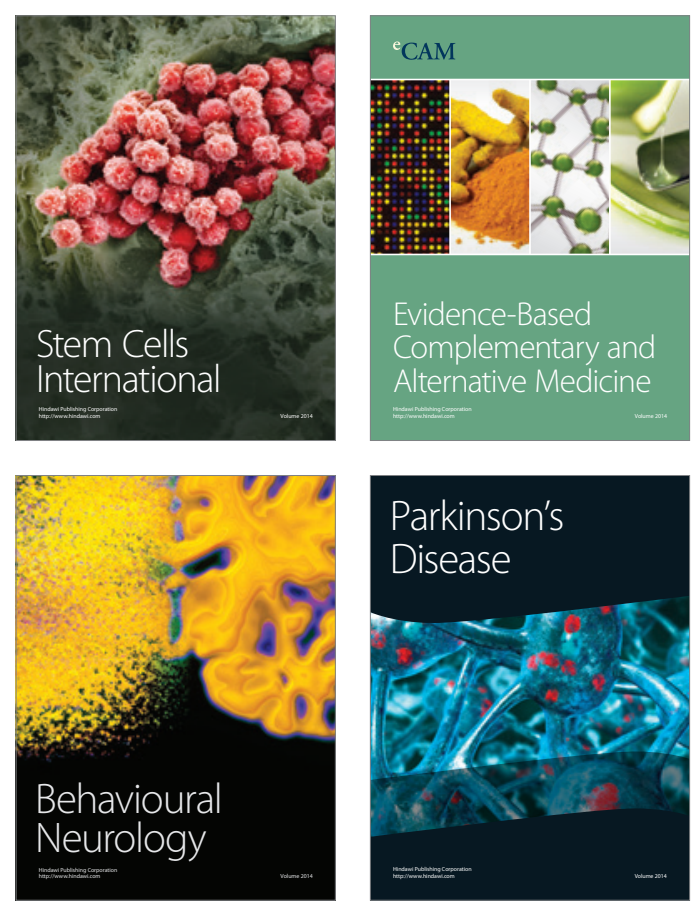

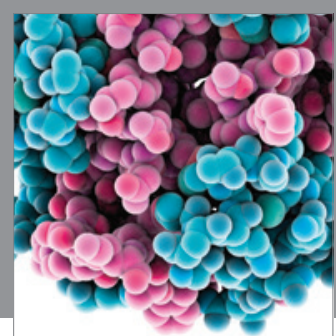

Journal of
Diabetes Research

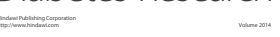

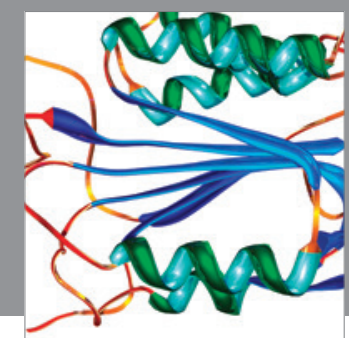

Disease Markers
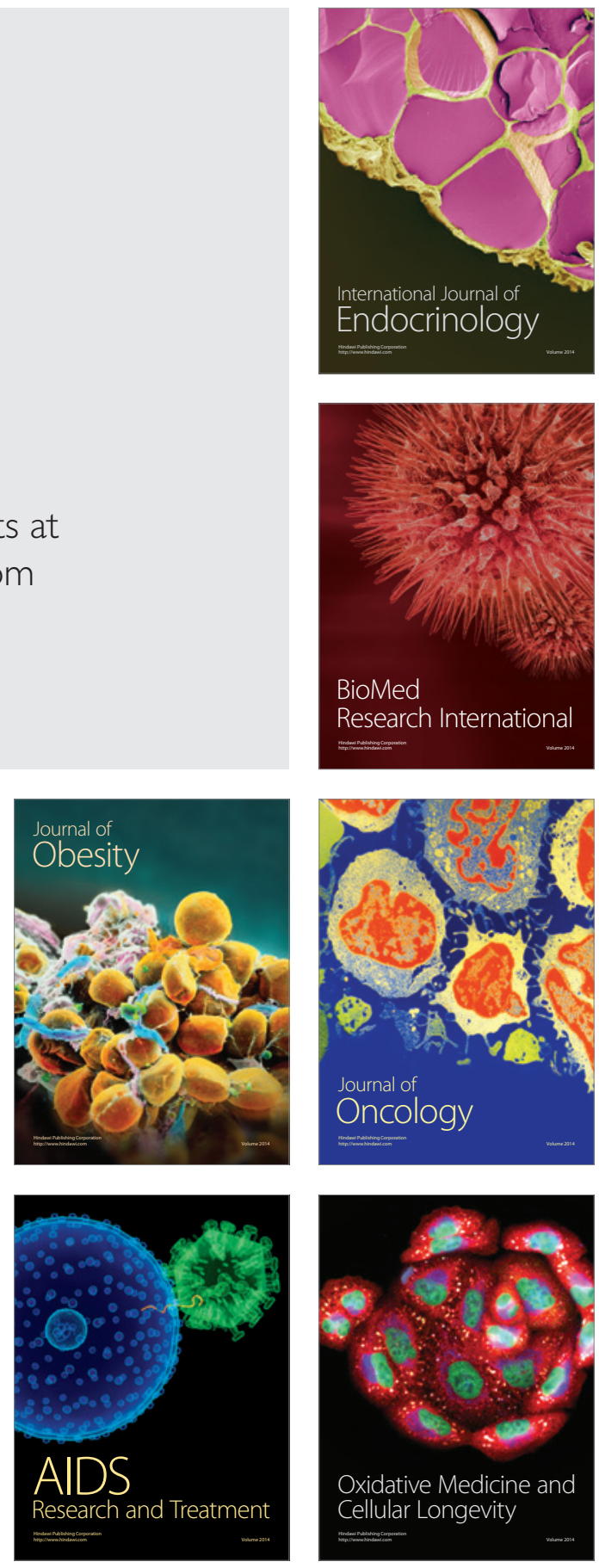